Canadian Art Review

\title{
RACAR
}

\section{Le design comme vecteur de changement social, les initiatives de Julien Hébert au Québec dans les années 1960 et 1970}

\section{Martin Racine}

Volume 40, numéro 2, 2015

Design Studies in Canada (and beyond) : The State of the Field Les études du design au Canada (et au-delà) : un état des lieux

URI : https://id.erudit.org/iderudit/1035397ar

DOI : https://doi.org/10.7202/1035397ar

Aller au sommaire du numéro

Éditeur(s)

UAAC-AAUC (University Art Association of Canada | Association d'art des universités du Canada)

ISSN

0315-9906 (imprimé)

1918-4778 (numérique)

Découvrir la revue

Citer cet article

Racine, M. (2015). Le design comme vecteur de changement social, les initiatives de Julien Hébert au Québec dans les années 1960 et 1970. RACAR: Revue d'art canadienne / Canadian Art Review, 40(2), 72-85.

https://doi.org/10.7202/1035397ar
Résumé de l'article

This article examines a lesser-known dimension of the career and work of Julien Hébert, one of the most influential figures in Quebec and Canadian design from the 1950s to the 1980s. By revisiting Hébert's archives, it sheds light on significant initiatives he developed in order to use design as a tool for cultural and social change. These are a project for a Montreal-based Institute of Design and another that aimed at tackling unemployment through design practice. These exemplify Hébert's understanding of design as a discipline that offers more than the creation of beautiful products and functional furniture. For him, it was a means to change society and to improve the wellbeing of its citizens.
Tous droits réservés (C) UAAC-AAUC (University Art Association of Canada | Association d'art des universités du Canada), 2015
Ce document est protégé par la loi sur le droit d'auteur. L’utilisation des services d'Érudit (y compris la reproduction) est assujettie à sa politique d'utilisation que vous pouvez consulter en ligne.

https://apropos.erudit.org/fr/usagers/politique-dutilisation/ 


\title{
Le design comme vecteur de changement social, les initiatives de Julien Hébert au Québec dans les années 1960 et 1970
}

\author{
Martin Racine
}

\begin{abstract}
This article examines a lesserknown dimension of the career and work of Julien Hébert, one of the most influential figures in Quebec and Canadian design from the 1950 s to the 1980 s. By revisiting Hébert's archives, it sheds light on significant initiatives he developed in order to use design as a tool for cultural and social change. These are a project for a Montreal-based Institute of Design and another that aimed at tackling unemployment through design practice. These exemplify Hébert's understanding of design as a discipline that offers more than the creation of beautiful products and functional furniture. For him, it was a means to change society and to improve the wellbeing of its citizens.
\end{abstract}

Martin Racine est professeur agrégé au Département de design et d'arts numériques de l'Université Concordia à Montréal.

-martin.racine@concordia.ca

1. Voir Martin Racine et Alain Findeli, «Julien Hébert and the Emergence of Industrial Design in Canada", Design Issues, vol. 29, $\mathrm{n}^{\circ} 4$, 2003, P. 31-43.

2. John Bruce Collins, Design for Use, Design for the Millions: Proposals and Options of the National Industrial Design Council of Canada, 1948-1960, Mémoire de maîtrise, Université Carleton, 1986
Nous avons déjà eu l'occasion de révéler le rôle de Julien Hébert (1917-1994) concernant l'émergence du design au Québec et au Canada, et il ne fait pas de doute que ce designer figure parmi les plus importants pionniers de la discipline au pays. ${ }^{1}$ Le présent article cherche à approfondir la pensée d'Hébert en ce qui a trait à l'impact potentiel du design sur les aspects sociaux, économiques et culturels du Québec. Dans l'objectif de mieux saisir l'originalité de la vision d'Hébert concernant le design et son apport potentiel à la société québécoise, nous avons puisé dans le fonds d'archives du designer déposé au Musée national des beaux-arts du Québec, où nous avons retracé les textes, discours et projets qu'il a échafaudés dans les années 1960 et 1970.

Suite à des études à l'École des beaux-arts de Montréal (1936-1941) puis en philosophie à l'Université de Montréal (licence en 1944), Hébert séjourne à Paris de 1946 à 1948 afin de poursuivre sa formation auprès du sculpteur cubiste Ossip Zadkine. De retour au pays il participe, en 1951, au premier concours de design à avoir lieu au Canada. Organisé par le Comité national de l'esthétique industrielle et coordonné par Donald Buchanan, alors directeur du Musée des beaux-arts du Canada à Ottawa, cet événement vise à favoriser la conversion de l'industrie militaire, qui avait émergé pendant la Seconde Guerre mondiale, vers l'industrie civile. Les organisateurs du concours s'inspirent d'initiatives semblables lancées par le Museum of Modern Art (MoMA) de New York, tel que le Organic Design in Home Furnishings organisé en 1940 qui visait à promouvoir le design moderne et avait récompensé les talents de Charles Eames et Eero Saarinen, menant ainsi au déploiement de leurs carrières en design. ${ }^{2}$ À la manière des initiatives du MoMA, les critères de sélection de la compétition canadienne sont fondés sur des principes modernistes qui cherchent à décourager l'ornementation dans le mobilier, mettant plutôt de l'avant l'usage innovateur du bois et de l'aluminium de même que l'exploitation de nouvelles technologies de mise en forme. C'est dans ce contexte qu'Hébert introduit un concept de fauteuil en tube d'aluminium supportant une toile tendue à la manière d'un siège-hamac. | fig. 1 | Une telle structure, alliant une forme épurée et la légèreté de l'aluminium, est tout à fait originale pour l'époque. En tant que finaliste du concours, la chaise d'Hébert est présentée dans les journaux canadiens, lui procurant une visibilité dans les médias qui fait en sorte qu'un important manufacturier de Montréal du nom de Sigmund Werner remarque l'ingéniosité du projet. Celui-ci fera dès lors appel aux services d'Hébert pour la création de mobilier d'extérieur au sein de son entreprise, la Sun-Lite. 

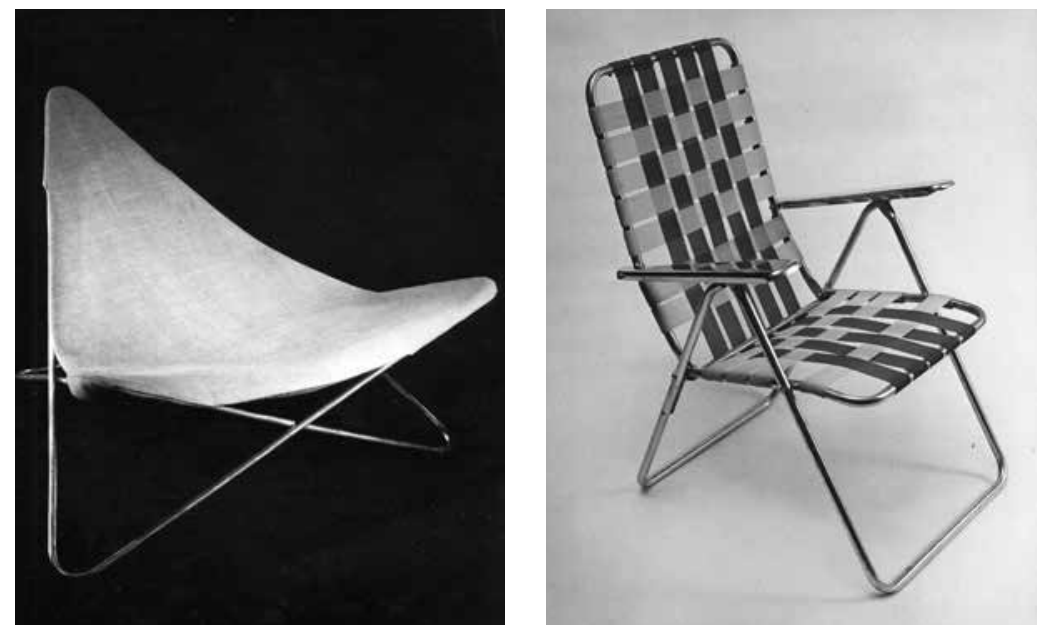

Figure $\mathbf{I}$ (gauche). Fauteuil conçu par Julien Hébert et soumis au premier concours de design au Canada en 1951. Photo d'archive du Fonds Julien Hébert, Musée national des beaux-arts du Québec, Québec, Bibliothèque et Centre de documentation.

Figure 2 (droite). Mobilier d'extérieur en aluminium dessiné par Julien Hébert et fabriqué par l'entreprise de Sigmund Werner à Montréal. Ce type de chaise pliante fut fabriqué à des dizaines de milliers d'exemplaires et distribué partout au Canada. Photo d'archive du Fonds Julien Hébert, Musée national des beaux-arts du Québec, Québec, Bibliothèque et Centre de documentation.

Du jour au lendemain, Hébert bifurque donc vers la carrière de designer industriel et se met à dessiner toute une gamme de mobilier en tube d'aluminium pour usage extérieur. | fig. 2 | Le succès lié à ses créations ne se fait pas attendre. Elles répondent à une demande dans les années 1950, à l'ère du développement des résidences de banlieue, pour un mobilier de jardin pratique, léger et confortable. Hébert deviendra ainsi au fil des années suivantes un designer multidisciplinaire reconnu au Québec et au Canada, de même qu'au niveau international. ${ }^{3}$

C'est ainsi grâce au concours et à ses retombées que le destin d'Hébert se définit: il sera designer. Cette nouvelle fonction lui apparaît comme une révélation, une réponse aux questions existentielles qu'il se pose sur la dimension sociale et l'utilité de l'art dans la société. En effet, Hébert se sent mal à l'aise face à la carrière de sculpteur qu'il avait pourtant entamée. Exposer ses œuvres dans une galerie dans le but de les vendre à une élite bourgeoise et aisée ne remplit pas ses aspirations sociales. Ses intérêts dans le milieu de l'art s'orientent d'ailleurs vers des créateurs qui arrivent à bâtir un pont entre la production artistique et l'environnement du quotidien. En outre, lors de son séjour en Europe, Hébert avait eu l'occasion de mieux connaître les artistes qui avaient gravité autour du Bauhaus (Johannes Itten, Wassily Kandinsky, Paul Klee, László Moholy-Nagy) et s'était intéressé aux valeurs prônées au sein de la célèbre école allemande fermée par les nazis en 1933, admirant la vision de Walter Gropius selon laquelle la hiérarchie entre les arts dits nobles et les arts appliqués devait être éliminée. Au Bauhaus, l'art et l'artisanat étaient mis sur un même niveau; en tournant le dos aux valeurs bourgeoises

3. Une des pièces de mobilier créées par Julien Hébert sera diffusée dans les revues de design internationales lors de sa présentation à la Triennale de Milan en 1954. Hébert participera à de nombreux événements internationaux au cours de sa carrière et sera invité comme professeur à l'Université de Californie à Los Angeles en 1964. et en intégrant toutes les disciplines de création, les praticiens pourraient rendre l'art-peinture, sculpture, design et architecture-accessible à tous. Au-delà de la dimension esthétique, le projet du Bauhaus était une entreprise globale visant à la fois l'aspect social et la transformation du cadre bâti, et autant les artistes que les designers et architectes y étaient impliqués.

Au début des années 1950, cette vision globalisante de l'art et du design intégré ne semble guère se manifester au Québec, alors dominé par la présence 
du clergé et les valeurs conservatrices. Ce qui ne veut pas dire que le milieu de l'art ne réagit pas à cette hégémonie, puisque comme on le sait, des groupes d'artistes menés par Alfred Pellan et Paul-Émile Borduas rédigent des manifestes qui remettent en question l'art traditionnel, l'idéologie dominante et l'emprise du clergé. ${ }^{4}$ Mais il faut reconnaître que ces groupes ne manifestent pas un intérêt marqué vers le design. Et Borduas enseigne pourtant à l'École du meuble. ${ }^{5}$ Pour Hébert, l'art appliqué, et donc le design, doit également faire partie du mouvement de renouveau artistique au même titre que la peinture ou la sculpture. Par sa vision intégrée des disciplines artistiques, Hébert se distingue nettement des courants de l'époque. Il tente même de promouvoir les idées du Bauhaus et d'introduire le design à l'École des beaux-arts de Montréal dès $1953 .{ }^{6} \mathrm{Il}$ ne remportera cependant pas de succès dans ses démarches, la direction de l'institution ne s'intéressant guère à cette discipline en émergence et tenant à rester fidèle à l'académisme classique.

\title{
L'éthique sociale de Julien Hébert
}

En 1978, jetant un regard rétrospectif sur sa carrière, Hébert écrit:

\begin{abstract}
Moholy-Nagy disait que le design n'est pas une profession mais une attitude. Le design est une attitude face à l'œuvre à faire qu'il s'agisse de grands ensembles ou de simples objets usuels. [...] La responsabilité du designer se situe non seulement au niveau du produit, mais aussi dans le contexte social, économique et culturel de notre société actuelle. ${ }^{7}$
\end{abstract}

4. Nous faisons référence au Manifeste "Prisme d'yeux», publié par Pellan en 1948 et au Manifeste du Refus global, également publié en 1948. Voir Guy Robert, La peinture au Québec depuis ses origines (Ottawa, 1978), p. 90-91, et Robert Bernier, Un siècle de peinture au Québec (Montréal, 1999), p. 200-201.

5. Pour un aperçu détaillé des visions divergentes de Borduas et Hébert, voir Martin Racine, "The Ambiguous Modernity of Julien Hébert», dans Expo'67: Not Just a Souvenir (Toronto, 2010), p. 93-108.

6. Selon une lettre de Julien Hébert adressée à la direction de l'École des beaux-arts de Montréal le 14 juin 1953. Fonds Julien Hébert, Musée national des beaux-arts du Québec (MNBAQ).

7. Texte de Julien Hébert intitulé «Le designer d'intérieur», non publié, Archives du Fonds Julien Hébert, MNBAQ, 28 février 1978.

8. La Commission Rioux se pencha sur la formation des diplômés dans les disciplines d'art et l'intégration de ceux-ci dans la société. Elle avait pour mandat d'étudier les questions relatives à l'enseignement des arts, y compris les structures administratives, l'organisation matérielle des institutions affectées à cet enseignement et la coordination de ces institutions avec les écoles de formation générale.
C'est avec ces mots à l'esprit qu'il faut aborder deux initiatives chères à Hébert: le projet d'un Institut de design à Montréal, proposé en 1961 et préparé à la demande du ministère de l'Industrie et du Commerce du Québec, et le Projet de réinsertion sociale, élaboré en 1971, et soumis quant à lui au ministère fédéral de l'Industrie et du Commerce. Dans le premier cas, le design est conçu comme étant à la base du développement culturel et économique de la société québécoise. Dans le second, il s'avère la source d'enjeux sociaux et devient un outil permettant aux laissés-pour-compte de reprendre un rôle actif au sein de la communauté. L'étude de ces deux propositions permet d'approfondir la vision d'Hébert quant aux responsabilités sociales et éthiques qu'il reconnaissait au design.

\section{Le contexte des années 1960 au Québec}

Suite à la mort de Maurice Duplessis en 1959 et à la prise de pouvoir par les Libéraux de Jean Lesage, la décennie des années 1960 marque un tournant majeur dans les structures sociales du Québec. Les réformes lancées dans les domaines de l'éducation et de la santé, jusqu'alors essentiellement dominés par les congrégations religieuses, créent un espace permettant aux valeurs humanistes et séculaires de prendre le relais vis-à-vis du clergé. Hébert adhère à cette mouvance de renouveau et de changement social: il participe, entre autres, aux audiences de la Commission Rioux sur l'enseignement des arts au Québec en 1966 et insiste pour que les principes de base du design soient intégrés au sein de l'enseignement des arts. ${ }^{8}$ II remarque d'ailleurs le retard que le Québec accuse dans ce domaine, et souhaite avant tout que le design soit une discipline reconnue en devenant une formation enseignée au niveau universitaire. Il regrette de voir le Québec négliger sa propre création de mobilier et cherche à développer et à rehausser la qualité de la production manufacturière afin de 
voir émerger un langage original et une signature propre au design québécois. Il est en ce sens très influencé par la qualité du design dans les pays scandinaves, en particulier le travail de l'architecte moderniste finlandais Alvar Aalto, chez lequel il décèle le signe d'une démarche inscrite dans un contexte culturel spécifique, reflétant un savoir-faire issu d'une longue tradition artisanale, notamment dans l'utilisation de matériaux tels le bois et le verre. Tout comme le design italien, français ou allemand, les créations scandinaves se démarquent, et c'est cette approche du design, reflétant une culture distincte et un langage esthétique particulier, qu'Hébert souhaite voir émerger au Québec.

\section{Le projet d'un Institut de design à Montréal}

Dans le préambule de son projet d'Institut de design, Hébert expose la situation du design au Québec et l'état pitoyable de la production industrielle de la province, qui, d'après lui, engendrent une absence d'identité et de qualité dans les produits québécois:

Copiés sur des modèles étrangers, ces produits sont désuets avant même d'être offerts en vente; créés à l'étranger et pour l'étranger, ils ne reflètent en rien notre habileté et nos goûts; dessinés pour une production qui n'est pas la nôtre, ils ne peuvent être produits dans nos usines à un prix de concurrence. ${ }^{9}$

Hébert souligne par ailleurs le manque d'initiative des Québécois concernant la transformation des ressources naturelles vers la production de biens manufacturés:

Un inventaire sommaire de nos ressources naturelles fait l'envie du monde; l'habileté
et l'audace des Québécois ne font pas de doute. Pourtant nos matières premières sont
exportées et nous reviennent fabriquées. Il y a là comme une démission massive du
peuple québécois. Il vit chichement de ses richesses naturelles au milieu de choses
modelées à l'étranger, selon un mode de vie qui n'est pas le sien. La plupart de nos
industries secondaires se contentent d'imiter plus ou moins bien un produit étranger. ${ }^{10}$

Hébert fait ainsi la promotion de la société québécoise comme ayant le potentiel de devenir une force tangible au niveau international en ce qui concerne la qualité des produits qu'elle peut développer. La résonance de ces propos, pris dans le contexte de 1961, est toute particulière. Indéniablement, la vision d'Hébert est en harmonie avec la dynamique de transformation et d'évolution sociale qui caractérise cette époque. Dans ce projet, il exprime en effet une foi profonde dans la société québécoise qui s'arrime d'emblée avec le célèbre slogan «maîtres chez nous» lancé par le gouvernement Lesage. Cette foi s'inscrit également de plain-pied au sein des réformes sociales, culturelles et écono-

9. Julien Hébert, Projet d'un Institut de design à Montréal, Mémoire déposé au ministère de l'Industrie et du Commerce, 1961, p. 4.

10. Ibid, p. 5 .

11. Le Deutscher Werkbund, fondé par Hermann Muthesius en 1907 à Munich, est une association d'industriels et d'artistes dont le but était de concilier industrie, modernité et esthétique afin d'améliorer la qualité de la production industrielle. Voir à ce sujet Frederic ) Schwartz, The Werkbund: Design Theory and Mass Culture Before the First World War (New Haven, 1996). miques amorcées au courant des années 1960. Lui qui a étudié dans un milieu d'avant-garde à Paris, rencontré les acteurs les plus influents du moment et voyagé à travers l'Europe, souhaite ardemment voir le Québec s'affirmer dans sa production manufacturière et développer des produits originaux qui seraient le reflet de sa culture propre.

Il est intéressant de faire un rapprochement historique entre le discours qu'Hébert déploie dans ce projet et celui de l'architecte allemand Hermann Muthesius, alors que ce dernier travaillait à la mise sur pied du Deutscher Werkbund en 1907 en Allemagne. ${ }^{11}$ L'objectif du Werkbund était précisément d'améliorer la qualité des produits fabriqués en Allemagne et de favoriser un partenariat entre les industriels et les artistes pour créer une esthétique 

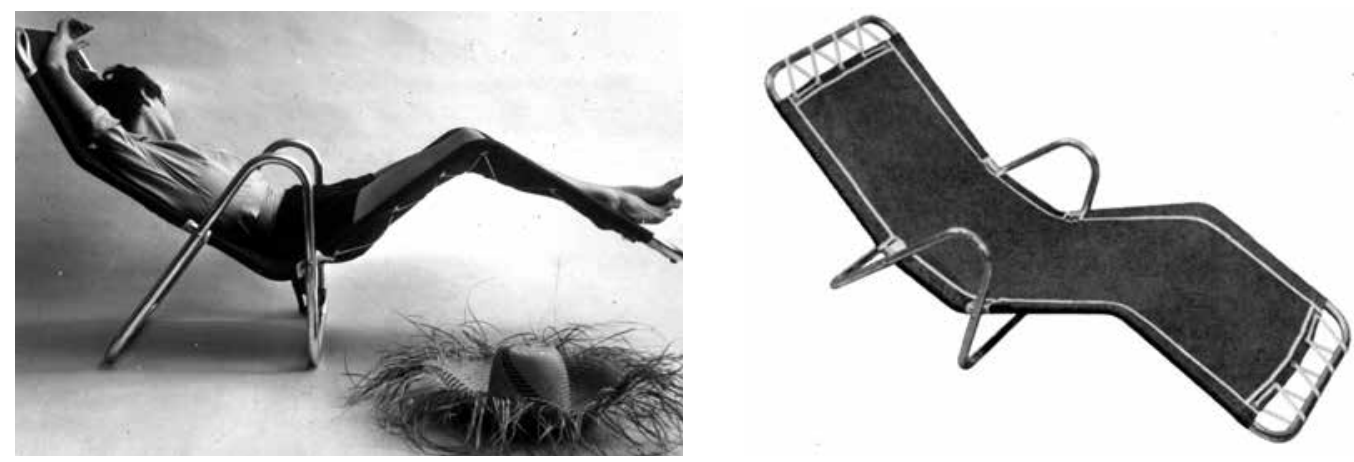

Figures 3 et 4 . Chaise longue en aluminium dessinée par Julien Hébert et présentée à la Triennale de Milan en 1954. Il s'agit de l'un des premiers exemples de design canadien à être reconnu et diffusé dans les revues de design internationales. Photo d'archive du Fonds Julien Hébert, Musée national des beaux-arts du Québec, Québec, Bibliothèque et Centre de documentation.
12. Alain Findeli, Le Bauhausde Chicago (Paris, 1995).

13. Jocelyn De Noblet, Design. Le geste et le compas (Paris, 1988).

14. Hébert, Projet d'un Institut, op. cit., p. 10.

15. Ibid, p. 8 . cohérente avec les principes de fabrication modernes, une initiative qui allait mener à la fondation du Bauhaus en 1919. C'est le même type de constat d'urgence qui avait motivé les membres de l'Association des arts et industries de Chicago à se tourner vers le design pour promouvoir la création industrielle au sein de sa production dans les années 1930:12 en effet, face à la piètre qualité des biens manufacturés dans la région, on avait fait appel à László MoholyNagy pour fonder le New Bauhaus à Chicago en 1937. Enfin, suite à la Deuxième Guerre mondiale, le Japan External Trade Organization (JETRO) avait été fondé au Japon pour stimuler le design pour des raisons semblables, alors que les produits japonais avaient une réputation de mauvaise qualité. ${ }^{13} \mathrm{La}$ pensée d'Hébert s'inscrit ainsi dans un contexte et une mouvance internationale qui cherche à favoriser l'essor du design sur le plan économique et pour stimuler une production cohérente, originale et de haut niveau.

Pour Hébert, cette volonté d'émancipation culturelle à travers le design, et économique à travers le développement de l'industrie secondaire de transformation, ne s'envisage pas en vase clos ou dans un esprit refermé sur luimême: «Le Québec a tout spécialement besoin d'être exposé aux arts et aux sciences du monde extérieur; il a tout avantage à être perméable aux grandes traditions internationales surtout en ce qui concerne l'esthétique industrielle». ${ }^{14}$ Par ailleurs, Hébert compte sur l'attrait de Montréal, qui selon lui est devenu par son site géographique un carrefour du monde, pour attirer les designers et pédagogues les plus compétents à l'Institut.

\section{La structure projetée de l'Institut et la formation des designers}

Hébert tient à une formation rigoureuse, de haut niveau, afin de voir le designer accéder au même statut que l'ingénieur, l'architecte, le chimiste et tout autre professionnel avec qui il serait appelé à collaborer. Il insiste d'ailleurs dans le mémoire déposé au ministère sur le fait qu'il est essentiel de ne pas se limiter à un simple cours d'initiation:

Produire des designers peu ou mal formés serait une erreur grave en ce moment et il faut à tout prix écarter la formule du compromis inspirée par une fausse prudence qui consisterait à créer un simple cours d'initiation à l'esthétique industrielle dans une école d'art, d'architecture ou de génie. Un tel cours d'initiation ne ferait que tromper tout le monde en commençant par les étudiants eux-mêmes. Il nous faut de toute urgence des designers qualifiés, des hommes mûrs [sic], capables de faire face aux conditions locales selon une pratique professionnelle égale, sinon supérieure, à celle des designers américains ou européens. ${ }^{15}$ 
Parce qu'il juge qu'il n'y a, ni au Québec ni au Canada, des professeurs capables d'assurer une formation professionnelle complète en design, il souhaite attirer des enseignants qualifiés d'Europe et des États-Unis. Ces praticiens et théoriciens pourraient alors inculquer leur expertise et permettre aux jeunes Québécois de découvrir les approches les plus innovantes en design tout en développant leur créativité et leur ingéniosité.

Dans les écrits d'Hébert, on dénote un sentiment d'urgence. En effet, il sent manifestement le retard à combler par rapport aux pays qu'il a visités: «Il nous faut immédiatement une école professionnelle de grande classe et il faut former immédiatement des professionnels de premier ordre. Pour parer à cette urgence et à cette difficulté, il me semble évident que la formation du designer doive commencer au niveau post-universitaire». ${ }^{16}$

Pour accélérer les choses, Hébert envisage effectivement de mettre sur pied une formation intensive de deux ans, du niveau de la maîtrise, pour laquelle les candidats seraient recrutés parmi les diplômés des écoles de génie, d'architecture ou d'autres écoles de même niveau. L'enseignement serait assuré par une équipe permanente de deux ou trois professeurs (dont implicitement il ferait partie), alors qu'une grande part des ateliers et séminaires seraient dirigés par des designers de réputation internationale, invités à passer quelques semaines à l'Institut. Par ailleurs, en créant cette formation d'études supérieures, Hébert prévoit également former des professeurs habiletés à enseigner le design au sein d'un département universitaire de premier cycle, qui n'existe toujours pas au Québec au début des années 1960. D’après lui, «Ce cours universitaire est urgent et devrait retenir immédiatement l'attention des autorités», ${ }^{17}$ mais il faudra attendre presque dix ans avant qu'une formation de premier cycle en design industriel voie le jour à l'Université de Montréal en 1969.

En ce qui concerne les projets qui seraient réalisés par les étudiants, Hébert exprime une vision pragmatique liée à son sentiment d'urgence. En effet, il tient à éviter que ceux-ci soient de simples exercices académiques et prône plutôt des travaux concrets répondant à des besoins précis de l'industrie québécoise. Il veut par exemple travailler en collaboration avec des entreprises telles que Bombardier, afin de permettre aux étudiants de découvrir le milieu manufacturier et d'obtenir une expérience réelle. Par ailleurs, il souhaite que les entreprises manufacturières établissent des liens de collaboration étroite avec l'Institut.

Hébert anticipe d'admettre une vingtaine d'étudiants dès l'ouverture et d'échelonner l'année scolaire sur cinquante-deux semaines pour utiliser pleinement les services de l'Institut et accélérer la formation des étudiants. La scolarité serait offerte gratuitement dans le but de favoriser un accès équitable à toutes les classes sociales; par ailleurs, dans un même esprit de démocratisation du savoir, les étudiants bénéficieraient de bourses généreuses pour se loger, en particulier ceux provenant de l'extérieur de Montréal.

\section{La promotion de l'esthétique industrielle}

Le deuxième volet de l'Institut serait voué à la promotion du design original, car plus que tout, Hébert a horreur de voir l'industrie québécoise imitant 
18. Ibid, p.13.

19. Ibid, p.14

20. Encore une fois, Julien Hébert se montre précurseur: aujourd'hui, il existe une revue américaine de design nommée ID. De son côté, l'idée d'un «Institut de design» à Montréal a finalement fait son chemin, mais presque quarante ans plus tard, puisque ce centre de promotion ne fut fondé qu'en 1996.

21. Hébert, Projet d'un Institut, op. cit., p. 32 québécois et fabriqués avec soin par l'industrie de la province. Il tient aussi à ce que les créations exposées répondent aux besoins locaux et qu'on en fasse la promotion au Québec et dans les marchés internationaux à travers des expositions itinérantes. Il souhaite par ailleurs que l'Institut devienne un espace de diffusion visant à sensibiliser le public québécois à la notion de design. Il envisage donc la promotion d'un type de production manufacturière distinctif et adapté aux besoins du marché québécois: «Le consommateur doit être informé, il doit être aussi tenté par le produit de qualité et être fier des produits dessinés et fabriqués dans le Québec».18

Pour arriver à ces objectifs d'éducation du grand public, Hébert planifie la mise sur pied d'un centre d'exposition dédié aux produits de qualité conçus et fabriqués au Québec. Sa vision est inspirée des centres de promotion du design qu'il avait étudiés et visités, hormis celui du Japon. Cinq modèles sont cités et analysés dans le projet présenté au ministère: le Design Centre à Londres (inauguré en 1956); le Svensk Form Design Centre à Stockholm (une association qui remonte à 1919); le Byggecentrum à Copenhague (ouvert en 1960); Den Permanente, également à Copenhague (un centre d'exposition permanente des arts décoratifs fondé en 1931); et le Japan Design House (mis sur pied en 1960, faisant partie du JETRO). Pour Hébert, ces centres soutenus par des fonds gouvernementaux jouent un rôle essentiel au déploiement et à la réputation du secteur du design dans ces différents pays. S'inspirant de ces exemples, Hébert veut ainsi faire la synthèse des meilleures stratégies nationales de promotion du design. Dans son texte, il déplore d'ailleurs le manque d'implication de l'État québécois pour favoriser le développement de ce secteur économique et cite le Japon en exemple, d'une manière qui étonne aujourd'hui, mais qui montre à quel point il était conscient, bien avant l'heure, de l'importance de mettre en place une structure de design visant la qualité: «Le Japon, copieur traditionnel et producteur à peu de frais à cause d'une main-d'œuvre mal payée, cherche par tous les moyens possibles à relever le niveau de sa production et tend vers la qualité». ${ }^{19}$ L'intervention du gouvernement japonais dans le secteur du design représente donc pour lui un modèle à suivre et il insiste pour préciser que "plus de soixante designers japonais étudiaient présentement le design aux frais de l'état dans différents pays».

À l'instar des centres visités, «l'Institut de design»— «l'ID», c'est ainsi qu'il veut le nommer pour ses consonances phonétiques ${ }^{20}$ - devrait se concentrer sur la promotion du design local:

Seuls les produits du Québec seront exposés au Centre, à moins qu'il soit jugé avantageux de faire certains échanges d'expositions avec l'étranger ou le National Design Council à Ottawa. Cette formule restrictive est en vigueur à Londres, Copenhague et Stockholm et me paraît nettement justifiable et dans l'intérêt de l'industrie du Québec. ${ }^{21}$

Dans cet extrait, Hébert décrit le Québec comme un État et se concentre strictement sur les besoins de l'industrie québécoise, sans faire mention de la situation au Canada. Il est permis de supposer qu'il est happé, comme plusieurs acteurs politiques et sociaux des années 1960, par une mouvance nationaliste qui cherche à valoriser l'État québécois et à affirmer le déploiement d'une identité culturelle distincte. Rappelons toutefois que le rapport est destiné au gouvernement provincial, ce qui teinte certainement le niveau et la stratégie de son intervention. 


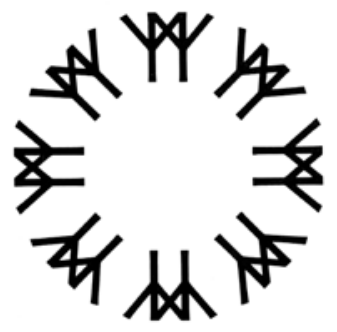

Figure 5. Symbole dessiné par Julien Hébert pour l'Expo 67 , représentant le thème de Saint-Exupéry, "Terre des hommes". Photo d'archive du Fonds Julien Hébert, Musée national des beaux-arts du Québec, Québec, Bibliothèque et Centre de documentation.
Hébert a certes une vision très ambitieuse pour l'Institut de design qu'il espère implanter à Montréal. Il le dépeint comme un espace ouvert, vivant, dynamique, dans lequel étudiants, dirigeants d'entreprise et grand public pourront converger, visiter des expositions, assister à des conférences et participer à des débats, ce qui permettrait au secteur du design de devenir un enjeu réel de développement économique et culturel au Québec. Dans sa conception, l'Institut abritera également un centre de documentation destiné autant aux étudiants qu'aux entrepreneurs, en plus de coordonner la publication d'une revue bilingue sur le design qui serait diffusée au niveau international. Des conférences et séminaires seront organisés régulièrement afin de contribuer au rayonnement des designers professionnels, ainsi que d'éduquer et démystifier le domaine du design auprès du public. Par ailleurs, il envisage la mise sur pied d'une association professionnelle de designers susceptible de collaborer au renouveau des arts industriels du Québec. Rappelons qu'il fera partie des fondateurs de l'Association des designers industriels du Québec en 1964. Finalement, Hébert prévoit également que l'Institut fera la promotion du design québécois à l'étranger en favorisant l'exportation des produits au niveau international. En somme, dans la vision d'Hébert, l'Institut serait un centre actif de formation et de promotion du design, octroyant des subventions de recherches, publiant une revue, décernant des prix et organisant des concours afin de faire accéder le Québec à des standards de qualité internationale dans le domaine de la création industrielle.

Il faut rappeler que, depuis le début des années 1950, Hébert a le vent dans les voiles et souhaite ardemment voir le domaine du design prendre de l'importance au Québec. Il est un des rares designers reconnus pendant cette époque et travaille sur des projets de mobilier, de luminaires, de graphisme, de design d'exposition, de murales intégrées à l'architecture (notamment à la Place des Arts en 1962 et au Centre national des arts à Ottawa en 1965) et réussit à enseigner la notion du design en devenant professeur, de 1956 à 1966, à l'École du meuble (renommée Institut des arts appliqués en 1958). Il poursuit activement, par ailleurs, sa pratique de designer. Au début des années 1960, Hébert se démarque en remportant le concours pour le dessin du symbole d'Expo 67, qui illustre le thème «Terre des hommes». Il réalise également la section des ressources naturelles du pavillon du Canada, ainsi que l'aménagement de sa cafétéria, pour laquelle il dessine le mobilier et la vaisselle. Soulignons la créativité d'Hébert dans ces projets: il privilégie en effet une relation formelle entre le symbole graphique de l'exposition et la structure du mobilier, comme si le dessin en aplat se déployait dans l'espace.

Malgré la notoriété d'Hébert, sa volonté et ses efforts soutenus, le projet d'Institut de design, déposé en décembre 1961, demeurera sans suite. Insensible à ses arguments, le ministère de l'Industrie et du Commerce du Québec ne se montre pas disposé à créer une telle institution. Il faut tout de même préciser que le domaine du design industriel est encore bien peu connu au Québec et que le secteur manufacturier demeure relativement peu développé en comparaison au domaine de l'industrie primaire liée à l'exploitation des ressources naturelles (mines, forêts, agriculture). Dans les années 1960, le gouvernement provincial met surtout de l'avant des structures permettant la croissance économique, notamment la Société générale de financement 

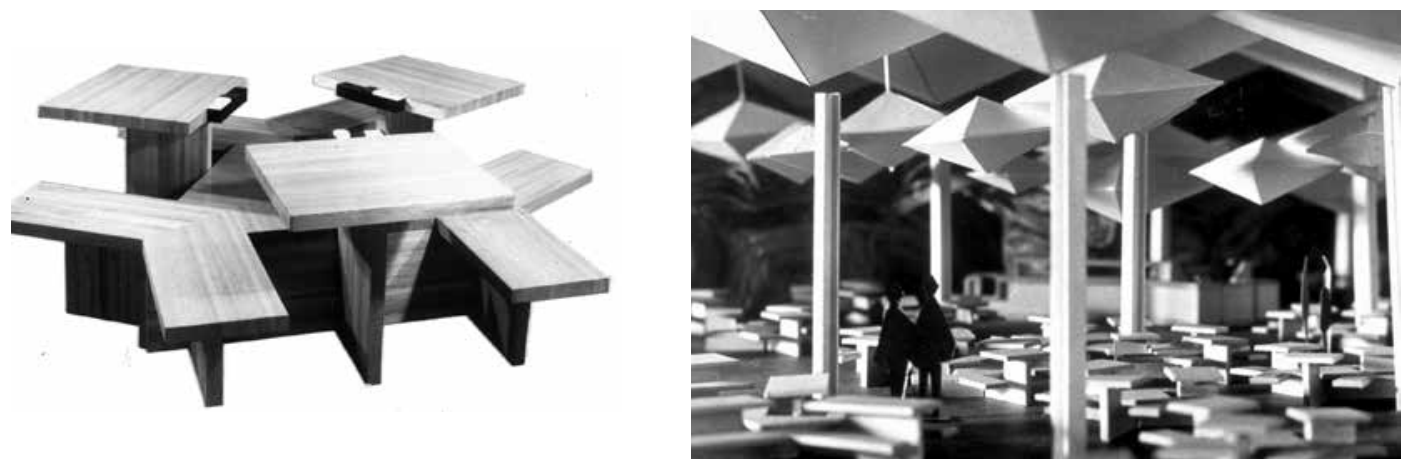

Figure 6. Mobilier pour la cafétéria du Pavillon du Canada à l'Expo 67, design de Julien Hébert. Photo d'archive du Fonds Julien Hébert, Musée national des beaux-arts du Québec, Québec, Bibliothèque et Centre de documentation.

Figure 7. Photo de la maquette de la cafétéria du Pavillon du Canada à l'Expo 67 , aménagement de Julien Hébert. Photo d'archive du Fonds Julien Hébert, Musée national des beaux-arts du Québec, Québec, Bibliothèque et Centre de documentation.
22. Rapport annuel du National Design Council 1974-1975, Ottawa, p. 3.

23. Julien Hébert, Projet de récupération de travail chez les déshérités. Document soumis au ministre de I'Industrie et du Commerce fédéral le 15 février 1971. en 1962 et la Caisse de dépôt et placement en 1965. Ce sont ces institutions qui vont permettre le développement et l'esprit d'entrepreneuriat dans les années 1970. Malgré ce contexte, le manque d'intérêt pour la promotion du design exprimé par le gouvernement Lesage dans les années 1960 ne fait que contribuer à retarder l'évolution du Québec en ce qui a trait à l'émergence du domaine du design au sein de l'industrie de transformation secondaire.

Bien que le gouvernement fédéral ait été plus favorable à la "cause», à travers des initiatives telles que les concours de design et la mise sur pied d'un Conseil national d'esthétique industrielle dans les années 1950, il reste que pour Hébert, ni le Québec ni le Canada n'accordent la place que le design mérite. Se contentant d'une économie basée sur l'exportation de ressources naturelles, le pays se limite à consommer des produits conçus et fabriqués à l'étranger.

D'ailleurs, cette situation n'évolue que très lentement. En 1974, Sonja Bata, directrice du National Design Council à Ottawa, écrit dans son rapport annuel:

Although we are capable of excellent design in Canada, our potential is not being fully realized. The vital and changing role of design and its impact on our society and economy are generally not appreciated. To a greater degree than would be considered normal for an industrialized country, Canada depends upon foreign sources for design. This detracts from our quality of life and inhibits technological, industrial and trade developments. ${ }^{22}$

\section{Le «Projet de récupération de travail chez les déshérités»}

En 1971, Hébert rédige un nouveau projet qu'il soumet au ministre de l'Industrie et du Commerce fédéral, Jean-Luc Pépin. Ses visées sont d'utiliser le design industriel en tant qu'outil de créativité et de développement social et économique. Hébert se penche cette fois plus précisément sur les gens qui sont sans emploi et qui demeurent isolés, en particulier dans les régions. «Il s'agit de récupérer pour le monde du travail des personnes qui désespèrent d'y participer. Ces personnes sont les déshérités que la société technologique n’absorbe pas et qui, par leur faute ou non, ne participent pas à l'abondance de notre société». ${ }^{23}$

Dans son texte, Hébert fait l'apologie du colon venu s'établir en Amérique, assumant son sort et sa destinée en toute liberté. L'auteur rappelle que les arrivants, autonomes et débrouillards, défrichaient et ensemençaient leurs terres, construisaient leurs maisons et se vêtaient avec leurs propres moyens. Selon lui, ils n'avaient rien à attendre d'un gouvernement et bâtissaient 
eux-mêmes leurs églises et leurs écoles. Hébert vante leur persévérance et la qualité de leurs entreprises; il se montre admiratif d'un tel esprit d'initiative. Il avance cependant qu'à mesure que les techniques avaient évolué et étaient devenues accessibles, l'esprit d'initiative et la volonté d'entreprendre s'étaient dissipés, en particulier auprès de la population moins favorisée:

Tout se passe comme si la technologie dépassait l'homme simple et l'abandonnait, sans foi ni courage, à l'arrière d'un progrès qui fait miroiter à ses yeux un mode de vie splendide, mais de moins en moins accessible. Travaux et entreprises semblent nécessiter tellement de connaissances scientifiques, de qualifications administratives, d'investissements et de manipulations légales et financières, qu'un brave homme [sic] doit d'avance s'avouer incapable et vaincu. L'emploi lui viendra de l'initiative des autres et sans emploi, l'argent viendra des sources de l'État.

Comment redonner à l'homme la confiance en sa propre initiative? Comment le remettre au point initial de l'entreprise et lui donner une force d'inertie suffisante? Comment redonner aux déshérités sans ressources, sans équipement, sans marchés, le sens et le goût de l'entreprise? ${ }^{24}$

Pour pallier la situation, Hébert propose la mise sur pied d'une entreprise, à échelle réduite, de production d'objets simples qui nécessite peu de matériaux et seulement quelques outils ordinaires. Il perçoit cette «mini-industrie» comme le commencement de la petite industrie et de la grande; comme le point de départ de l'homme entreprenant qui, même sans ressources, veut produire à l'aide de ses dix doigts et de sa bonne volonté. Hébert se défend toutefois de suggérer un travail de type artisanal:

\footnotetext{
Mini-industrie ne veut pas dire artisanat. L'artisanat, au sens où on l'entend au Canada, se situe, d'une façon obstinée, en marge de l'industrie et encourage le "fait main» et le style "grand-mère». Cette conception vieillotte et sentimentale de l'artisanat a distrait presque tous les artisans de leur mission de créateurs et d'industriels engagés dans la société contemporaine. ${ }^{25}$
}

Au contraire, la vision d'Hébert est tournée vers l'avenir. À l'instar des fondateurs du Bauhaus qui l'avaient tant inspiré, il présente "l'industrie» comme quelque chose de noble, une institution moderne, inscrite dans le présent et l'avenir, dont le design forme l'assise et la fondation. En effet, lorsqu'il présente l'exemple de l'entreprise Bombardier, il précise qu'à la base de cette compagnie, il y avait une idée, un design, développé par un homme entrepreneur, une référence à l'œuvre de Joseph-Armand Bombardier, qui avait inventé l'autoneige en 1939. Pour Hébert, le besoin de concevoir un véhicule adapté aux conditions de routes hivernales et répondant aux nécessités locales est l'exemple parfait d'une démarche inscrite au sein d'un environnement physique et culturel donné, où fleurissent l'ingéniosité et la créativité. En suggérant l'idée d'une mini-industrie, Hébert veut recréer, avec des moyens modestes, la fierté d'accomplir un produit utile et de qualité répondant aux désirs des populations locales. Il exprime ainsi une vision idéalisée du travail, selon laquelle l'industrie est la voie à suivre pour émanciper le travailleur, faisant fi de toutes les misères que l'on associe souvent au travail en usine, de ses bas salaires et des tâches souvent ardues et répétitives dans un environnement malsain. «L'objet du présent projet est avant tout de faire produire, de plonger l'homme inoccupé dans une entreprise, si limitée soit-elle, afin qu'il sorte de son oisiveté, qu'il se sente engagé dans une entreprise personnelle et qu'il retrouve la dignité du travail»», ${ }^{26}$ 
Hébert suggère qu'il y ait plusieurs équipes de designers impliquées dans ce projet, afin qu'ils conçoivent différents objets à produire. Il propose donc la formation d'une équipe volante de jeunes designers capables de faire la promotion du programme dans des régions déterminées et qui s'attarderaient à développer une production de concepts et de prototypes liés aux besoins locaux. Toutefois, ceux-ci ne seraient que la bougie d'allumage: une fois la mini-industrie rodée, les travailleurs en place seraient encouragés à prendre des initiatives et à proposer leurs propres projets, avec la collaboration de designers.

Hébert envisage trois étapes. Tout d'abord, un projet pilote sera lancé dans une région où l'on retrouve un grand nombre de "déshérités» (des gens au chômage ou des assistés sociaux). Il s'agit de fabriquer des objets faciles à assembler dans un matériau acquis à peu de frais. Ces produits devront comporter des possibilités de mise en forme systématisée, de façon à stimuler l'initiative de l'exécutant en l'entraînant à la réalisation en série, même si celle-ci est limitée à quelques exemplaires. Les modèles développés seront ingénieux, stimulants, piquant la curiosité, comme des casse-tête, des jeux ou des objets usuels apportant une solution inattendue à un besoin. En plus de faciliter la vente des produits, cela stimulera le fabricant à prendre plaisir à créer quelque chose d'innovateur. Les objets devront avoir une telle qualité esthétique qu'ils pourront être introduits sur les marchés les plus sophistiqués et donner à «l'industriel» la juste fierté de son produit. En second lieu, on analysera les résultats obtenus, corrigera les failles et envisagera un plus grand nombre de produits plus complexes, fabriqués dans une variété de matériaux: bois, verre, aluminium, etc. Les thèmes pour cette production pourront être variés et non plus limités aux jeux ou casse-tête. Hébert suggère d'organiser des concours pour les nouveaux biens à développer et de prévoir des récompenses pour la qualité de la fabrication. La troisième étape anticipée demeure fort ambitieuse, il s'agit de laisser croître les initiatives locales et d'encourager la transformation de ces "mini-industries» en petites industries et même, si cela est possible, en grandes industries, en aidant à fournir la machinerie nécessaire à l'élargissement de l'entreprise.

On ne peut qu'être frappé par la vision utopique, presque naïve, proposée par Hébert en parcourant les quelque vingt pages qui décrivent ce projet. Il ne s'agit pourtant pas d'une vision idéalisée selon Hébert, qui se défend de mettre sur pied une «énième» entreprise de sauvetage à l'image de dizaines d'autres programmes d'assistance gouvernementaux. Il se montre au contraire convaincu que la proposition qu'il soumet saura raviver l'esprit d'initiative des individus isolés, en particulier ceux des régions défavorisées, loin des centres urbains-il pense également à l'intégration des anciens détenus, des gens âgés, des handicapés physiques ou mentaux, etc.

La position d'Hébert ne constitue pourtant pas une simple apologie de l'industrie ni un plan simpliste de réinsertion dans les milieux du travail. En effet, son désir n'est pas de «mettre les assistés au travail»à tout prix, dans des tâches ingrates s'il le faut. Au contraire, il se montre critique vis-à-vis d'un type d'industrie déshumanisant où l'esprit d'initiative estabsent. C'est la notion de créativité au sein de l'industrie, de même que la prise en charge des besoins locaux et l'indépendance économique que celle-ci peut générer qui intéressent 
Hébert. Ces éléments sont des critères intrinsèques à sa vision globale et à son éthique du design. Le design industriel, en tant qu'outil de créativité, à la source de la production, joue ainsi un rôle social dont la finalité demeure l'être humain et sa dignité. Comme le designer l'explique dans son projet:

En créant une société à l'image d'un Brave New World, nous entraînons une partie de la masse dans une aventure qui ne lui est pas favorable et nous nous imposons, d'autre part, des responsabilités et des opérations de sauvetage telles que des dizaines de programmes d'assistance de nos gouvernements. Le présent projet ne veut pas être un autre programme d'assistance, mais au contraire un moyen d'éviter quelques-uns de ces programmes en les rendant inutiles. Il a peut-être un caractère utopique en ce sens qu'il suppose l'existence chez les hommes d'un désir réel de survie. ${ }^{27}$

Hébert restera sans nouvelles de ce projet. Un an après avoir envoyé sa proposition, il achemine une lettre au ministère indiquant vouloir récupérer son texte puisqu'il n'y a pas eu de suites. Confus, le chef du cabinet rappelle Hébert pour lui dire que le document est introuvable. Il lui assure pourtant que le ministre l'avait lu...

\section{Conclusion-Julien Hébert: un designer humaniste}

Ces deux projets, l'un basé sur un programme pédagogique, l'autre visant le réemploi, témoignent de la vision globalisante qu'Hébert avait du design. Dépassant la simple conception de produits et de mobilier, le design représentait pour lui une activité destinée à l'amélioration de la société dans son ensemble, contribuant à son essor économique, culturel et social. Hébert avait de grandes ambitions pour le Québec. En désirant doter Montréal d'un institut de niveau international, il souhaitait voir émerger une culture du design forte, originale et distinctive. Il comptait former des designers capables de créer des produits de qualité, dont la valeur serait reconnue à l'étranger et qui pourraient ainsi être exportés. Dans son projet pour les «déshérités", il exprimait une vision humaniste, inspirée de la pensée du philosophe thomiste Jacques Maritain. ${ }^{28}$ Par ailleurs, l'action d'Hébert s'accordait avec celles d'autres intellectuels de sa génération, tels Pierre Trudeau, René Lévesque ou Jean Marchand, qui exprimaient une volonté de progrès social. Alors que ces derniers embrassaient le syndicalisme et la politique comme lieu d'action sociale, Hébert s'affirmait en tant que créateur et pédagogue et voyait dans le design un outil susceptible de développer la société et de transmettre des valeurs d'entraide.

Pourtant, sa volonté et sa passion pour le design se heurtaient à un mur dans son propre pays. Il acceptait mal l'état de la situation et ne pouvait se résoudre à voir les choses stagner dans la médiocrité. Dans une allocution prononcée en 1962, Hébert déclarait dans un esprit de critique acerbe:

27. Ibid, p. 12. Les mots sont soulignés par Hébert.

28. Pour ce philosophe, il demeurait essentiel d'aller au-delà de l'action «en tant que chrétien» (une action limitée à l'obéissance aux rites et aux dogmes de l'Église), pour agir "en chrétien», à travers la mise en œuvre, individuellement, des idées chrétiennes dans le domaine public. Voir Jacques Maritain, Art et scolastique (Paris, 1920).
L'esthétique industrielle est à l'origine du produit. Mais, dans notre industrie, le produit est le plus souvent enfant naturel. Il naît au hasard de l'entreprise et l'entreprise le porte sans fierté, mais pour le seul profit qu'elle peut en tirer.

Faut-il s'étonner que les ouvriers d'une usine ne pensent qu'à leur sécurité, ou que le fondateur d'une usine l'abandonne dès qu'il en a tiré quelques centaines de milliers de dollars et lui préfère la Floride? Le produit n'y est pas. On fabrique quelque chose qui ne vaut pas la peine d'être fabriqué. Quelque chose qu'il faut modifier tous les ans ou tous les six mois non pas pour l'améliorer mais surtout pour faire oublier la monstruosité produite antérieurement. Et la publicité et le commerce se chargent de faire accepter ce n'importe quoi qu'est le produit à des consommateurs bien endormis. 
Il n'est peut-être pas de mise dans le monde de l'industrie et du commerce de faire la morale ou de parler du bien-être et du bonheur des hommes mais il me semble que presque tout ce que produit l'industrie du Québec a un pouvoir avilissant. Un humoriste disait qu'il est bien d'avoir les pieds sur terre mais qu'il n'est pas nécessaire de s'y enfoncer comme un ver.

Je ne doute pas que le produit sans qualité ni valeur trouve son marché chez nous mais je m'étonne et n'accepte pas que notre industrie se contente de ce marché et s'y complaise alors même que les produits de qualité trouvent ici même un marché considérable et que d'autre part existent à l'étranger des marchés illimités pour des produits qui pourraient les satisfaire. ${ }^{29}$

Pour Hébert, toute entreprise industrielle ne pouvait prendre sa valeur qu'à travers l'excellence du design de ses produits comme, par exemple, dans les pays scandinaves ou en Italie. Pour lui, ces cultures avaient le mérite d'avoir réussi, non pas une cassure nette entre l'artisanat et l'industrie, mais plutôt l'intégration harmonieuse de l'un dans l'autre. Le goût du travail bien fait, si présent dans la tradition artisanale, pouvait très bien s'exprimer de la même manière au sein de l'industrie et de la technologie moderne. C'est d'ailleurs ce qu'il décrivait en 1962:

Ce ne sont pas les exigences du marché italien qui ont forcé Olivetti à bien dessiner ses machines. Le goût de la masse des acheteurs en Italie n'a pas cette puissance discriminatoire. Mais Olivetti a cru en ce qu'il faisait suffisamment pour bien le faire et il en est résulté un produit de qualité qui a conquis les Italiens et le monde entier. ${ }^{30}$

Quant à la société québécoise, Hébert y percevait un énorme potentiel. Ses attentes étaient d'autant plus exigeantes qu'il ne voyait pas pourquoi le Québec ne figurerait pas parmi les leaders mondiaux en design. La longue citation qui suit permet de bien saisir sa passion pour le développement du design et sa déception face au retard du Québec dans ce domaine:

\begin{abstract}
Nous avons dans le Québec les matières premières, les sources d'énergie, une maind'œuvre habile et douée, des capitaux limités mais qui dorment le plus souvent. Nous avons l'espace, le goût de l'aventure, du risque, une certaine âpreté au gain, une fierté presque excessive, une originalité certaine, le goût de la liberté jusqu'à l'autonomie. Nous avons un marché local restreint peut-être mais qui intéresse malgré tout les fabricants étrangers. Nous avons des amis puissants: les États-Unis, l'Angleterre et la France. Et si nous affichions un certain pacifisme international le reste du monde serait avec nous. Quel peuple peut prétendre à tant d'avantages? Et pourtant cette abondance de forces, de talents, de relations et de privilèges n'a pas réussi à faire du Québec une puissance économique de quelque importance au niveau de l'industrie secondaire. Nous sommes les artisans miteux, d'une camelote méprisable qui réussit encore à se vendre tant bien que mal parce qu'au niveau de son commerce tout se vend.
\end{abstract}

Peut-on faire une liste des objets manufacturés dans le Québec dont nous sommes fiers, j'entends des objets qui ont leur origine ici et qui ne sont pas des copies de quelque chose de l'étranger? Non seulement nous ne pouvons pas être fiers de ce que nous produisons mais nous ne produisons qu'une faible partie de ce que nous utilisons. L'équipement de ma maison, de mon bureau, de l'école où j'enseigne est en grande partie d'origine étrangère. Et je dois ajouter, ce sont les objets de qualité qui sont de l'étranger. ${ }^{31}$

29. Texte d'une allocution présentée à l'Université de Montréal en 1962. Fonds d'archives de Julien Hébert, MNBAQ.

30. Ibid.

31. Ibid.
Le point de vue d'Hébert demeure ici très critique, mais il faut admettre que le designer a travaillé toute sa vie dans l'objectif de changer les choses et de développer une vision du design spécifique au Québec. À travers ses efforts pour la mise en place de projets structurants, Hébert a cherché à ancrer ceuxci dans le contexte et la réalité québécoise, faisant référence à son histoire et cherchant à revaloriser le rôle des bâtisseurs qui ont permis son évolution. Sa vision du design ne se limitait pas au niveau d'un outil de rentabilité pour les 
32. Jean-Claude Marsan, Julien Hébert, prix Borduas 1979, http:// www.prixduquebec.gouv.qc.ca/ recherche/desclaureat.php?noLaureat $=179$. entreprises. Elle transcendait l'aspect économique pour accéder à un niveau plus idéaliste, où la discipline ferait évoluer le Québec vers une plus grande émancipation culturelle, tout en favorisant une plus grande justice sociale. Malgré l'échec des initiatives élaborées par Hébert au courant des années 1960 et 1970, il faut rappeler qu'Hébert a eu l'occasion de transmettre sa vision du design en contribuant à former plusieurs générations de designers à travers son rôle d'enseignant, d'abord à l'Institut des arts appliqués, puis à l'École de design industriel de l'Université de Montréal, une école de design qu'il a largement contribué à fonder. Ce sont par ailleurs le caractère humaniste et la vision sociale d'Hébert qui ont été soulignés lors de la remise du prix Borduas au designer en 1979 pour saluer l'excellence de sa carrière de créateur. Rappelons que ce prix, reconnu pour être la plus haute distinction en arts visuels au Québec, couronnait pour la première fois l'œuvre d'un designer. Dans le discours qu'il prononce pour présenter Hébert, le recteur de la Faculté de l'aménagement de l'Université de Montréal, Jean-Claude Marsan, alors président du jury déclare:

Julien Hébert a été un pionnier de la pratique et de l'enseignement du design industriel, et sa carrière se confond avec le développement de cette discipline au Québec, auquel il contribua plus que tout autre, même s'il se refuse par modestie à l'admettre. L'activité de Julien Hébert s'est exercée dans toutes les directions, ce qui est dans l'ordre des choses puisque le designer n'est pas un artiste comme les autres. Sa discipline n'a rien à voir avec l'ego et les états d'âme; il n'exprime pas son moi et cherche ni à se libérer ni à convaincre. Il répond essentiellement aux besoins des autres. ${ }^{32}$ 